\title{
Editorial
}

\section{LÍNEAS DE INVESTIGACIÓN EN ODONTOLOGÍA}

Una línea de investigación es un conjunto de temas que se articulan entre sí para hacer avanzar una parte de la ciencia. Esta agrupación es beneficiosa cuando permite la gestión del conocimiento, ayuda al desarrollo de la ciencia y cuando facilita la integración de procesos para la generación de nuevos conocimientos.

La Organización de Naciones Unidas para la Educación, la Ciencia y la Cultura (UNESCO), en 1978 realiza una serie de recomendaciones sobre la normalización internacional de las estadísticas relativas a la ciencia y tecnología, entre ellas conceptualiza las Actividades Científicas y Tecnológicas y además indica que dentro de estas, se consideran a la enseñanza y la formación científica y técnica. Clasifica las áreas de investigación en Ciencias Naturales, Ingeniería y Tecnología, Ciencias Médicas, Ciencias Agrícolas, Ciencias Sociales y Humanidades. Dentro de las C. Médicas existen tres sub áreas: Medicina básica, Medicina clínica (las especialidades de la medicina y la odontología) y Ciencias de la Salud. En la actualidad el paradigma que sostiene a las Ciencias médicas se denomina medicina basada en evidencias, el cual en concordancia con los diseños de estudios clínicos nos deja 5 líneas de investigación que se pueden aplicar a cualquiera de las especialidades de la Medicina clínica. En el caso de la odontología, son líneas de investigación:

1. Los factores de riesgo y su manejo en las enfermedades odonto estomatológicas.

2. La frecuencia de las enfermedades odonto estomatológicas.

3. Los métodos de diagnóstico y su valoración para las enfermedades odonto estomatológicas.

4. La efectividad de los tratamientos para las enfermedades odonto estomatológicas.

5. El pronóstico de las enfermedades odonto estomatológicas.

Teniendo como sexto línea de investigación adicional y compartida con las Ciencias de la Salud a la gestión y manejo de tecnologías en los servicios de salud.

El producto de todas estas líneas organizadas como un solo cuerpo, se sintetiza en un documento denominado Guía de Práctica Clínica, siendo esta la finalidad práctica de la investigación, dado que repercute en la comunidad mejorando el conocimiento que será aplicado para solucionar problemas de salud. Respecto a los problemas de salud bucal o necesidades de atención que deben ser estudiados por la Odontología, existe una clasificación propuesta en el artículo: 
"Taxonomy for competency-based dental curricula" ${ }^{1}$ planteadas por Roberto Beltrán-Neira en el Journal of Dental Education en 2004; que indica que se agrupan en:

a.- Problemas de Tejidos duros. (Ej.Caries, Lesiones no caries,Anomalías de forma)

b.- Problemas de Tejidos blandos.( Ej. Enf. Periodontal, Tumoraciones, Lesiones)

c.- Problemas de la Oclusión.(Ej. Malposición, edentulismo)

d.- Problemas de orden Médico.(Ej. Diabetes, Síndrome de Down, Automedicación)

e.- Problemas de orden Quirúrgico.(Ej. Labio y Paladar Fisurado, 3er molar retenidos)

f.- Problemas de orden Social. (Ej. Calidad de Vida, Hábitos, Autismo, Ansiedad).

Las líneas de investigación, no deben entenderse como parámetros rígidos, pues existe la investigación traslacional que lleva adelantos de un área de la ciencia a otra y genera tecnología, deben entenderse como una guía para organizar el avance de una parte de la ciencia. Desde la Universidad Católica de Cuenca- Ecuador, proponemos estas líneas de investigación Odontológica, e invitamos a la colaboración en nuestra revista a fin de incrementar el conocimiento en la profesión para el beneficio de la sociedad.

Dr. Ebingen Villavicencio Caparó

Director de la Revista

\footnotetext{
${ }^{1}$ Beltrán-Neira,R;Beltrán-Aguilar,E.Taxonomy for Competency-Based Dental Curricula. Journal of Dental Education; 2004 68(9):978-84
} 